\title{
Surgical Management of Chronic Unremitting Constipation and Fecal Incontinence Associated With Megarectum: A Preliminary Report
}

\author{
By Steven L. Lee, Jeffrey J. DuBois, Ramon G. Montes-Garces, Kristen Inglis, and William Biediger \\ Sacramento, California and Travis Air Force Base, California
}

\begin{abstract}
Background/Purpose: Functional constipation with associated fecal incontinence responds poorly to medical management once megarectum has developed. The authors describe resecting the dilated rectum and inserting a cecostomy button for antegrade enemas in this difficult condition.

Methods: Four children, ages 9 to 15 years, with a history of unremitting constipation and fecal incontinence were referred for evaluation after not responding to medical management. All patients had exhibited normal lumbosacral magnetic resonance images (MRI) and open rectal biopsies; however, all 4 patients had a megarectum on contrast enema. In addition, anorectal manometry was consistent with functional fecal retention. The dilated rectum was resected by anastamosing the nondilated sigmoid colon to the distal rectum, and a standard gastrostomy button was inserted into the cecum for antegrade enemas. Mean follow-up was 35 months (range, 8 to 60 months).
\end{abstract}

Results: Constipation and incontinence resolved within 6 months in all patients, and all children remained continent without the aid of cathartic agents. There were no postoperative episodes of fecal impaction. The only complication was antibiotic-associated diarrhea in 1 patient. Cecostomy buttons were removed at 1 year postplacement in all 4 patients with continued success. Three patients underwent repeat anorectal manometry; all 3 had normal rectal sensory threshold volumes and anorectal inhibitory reflexes. Barium studies also were obtained in the 3 patients without evidence of recurrent rectal dilation.

Conclusions: Refractory constipation and incontinence associated with megarectum may be amenable to surgical intervention in selected patients. The authors' limited experience suggests that proctectomy and button cecostomy is an effective treatment option that improves the quality of life in these patients. Furthermore, proctectomy alone may be curative.

$J$ Pediatr Surg 37:76-79. This is a US government work. There are no restrictions on its use.

INDEX WORDS: Megarectum, intractable constipation, segmental resection.
C HRONIC CHILDHOOD CONSTIPATION is a debilitating disease that is best managed by using a multidisciplinary approach. Primary care of these patients usually is managed by pediatricians and pediatric gastroenterologists and typically consists of diet modification, cathartic agents, enemas, biofeedback training, and psychotherapy. However, as many as $40 \%$ to $50 \%$ of such patients fail to respond to medical treatment, and surgical intervention has played an increasing role over the past decade in the management of functional constipation. ${ }^{1,2}$ In the past, pediatric surgeons were primarily limited to the treatment of the complications associated

From the Department of Surgery, University of California, Davis Health System, Sacramento, CA, and the Division of Pediatric Surgery and Gastroenterology, David Grant USAF Medical Center, Travis Air Force Base, CA.

Presented at the 31st Annual Meeting of the American Pediatric Surgery Association, Orlando, Florida, May 25 to May 28, 2000.

Address reprint requests to Jeffrey J. DuBois, MD, Department of Surgery, Division of Pediatric Surgery, 5275 "F" Street, Suite 3, Sacramento, CA 95819 .

This is a US government work. There are no restrictions on its use. 0022-3468/02/3701-0015\$00.00/0

doi:10.1053/jpsu.2002.29431 with constipation and the correction of congenital anorectal anomalies. Recently, this limited role has expanded, and earlier surgical intervention including transanal rectal biopsy, posterior internal sphincter myomectomy, and establishing access for antegrade enemas is undertaken for both evaluation and treatment of unremitting, chronic constipation. ${ }^{3-6}$

Intractable constipation and fecal incontinence also complicates postsurgical correction of anorectal malformations and may be caused by segmental dilation of the sigmoid colon or rectum. Many investigators have thus described curative resection of the megasigmoid or the atonic baggy rectum with good long-term results.7,8 Based on separate reports using segmental resection and antegrade enemas, we treated 4 children who had chronic, functional fecal retention and associated megarectum by combining these 2 methods. This report describes our technique and the results of resection of the dilated rectum and insertion of a cecostomy button for antegrade enemas.

\section{MATERIALS AND METHODS}

Four patients, ages 9 to 15 years, with a history of long-standing constipation and incontinence were referred for evaluation and man- 
agement after not responding to medical treatment. Despite prolonged treatment with diet modification, laxatives, stool softeners, and enemas, these patients suffered from repeated episodes of massive fecal impaction requiring multiple admissions for manual disimpaction. Magnetic resonance imaging (MRI) studies showed a normal lumbosacral cord, a normal number of sacral vertebrae, and central placement of the rectum in the levator sling in each child. All patients also had open rectal biopsy results showing normal ganglion cells present in the distal rectum. However, barium enema results showed a dilated megarectum (Fig 1) in all 4 patients. In addition, anorectal manometry was consistent with functional fecal retention, including impaired rectal sensation to balloon distention (threshold volumes were $>120 \mathrm{~mL}$, normal, 15 $\mathrm{mL}$ ) and a depressed but present anorectal inhibitory reflex.

Encouraged by reports that showed successful outcomes after segmental resection of dilated, atonic colon in patients with anorectal anomalies, and separate reports of antegrade enemas, we elected to perform resection of the megarectum and place a cecostomy button in these patients. ${ }^{4,5,7,8}$ Before operation, each patient underwent extensive preoperative bowel preparation including manual fecal disimpaction, gastrointestinal lavage with polyethylene glycol solution (GoLYTELY; Braintree Labs, Braintree, MA), and Fleet enemas. Preoperative systemic antibiotics also were administered.

In all patients, the rectum was extremely dilated, and the bowel wall consisted of very hypertrophied muscularis propria. To minimize the risk of damage to the pelvic nerves, the dissection of the rectum was maintained along its hypertrophied wall and carried down to approxi-

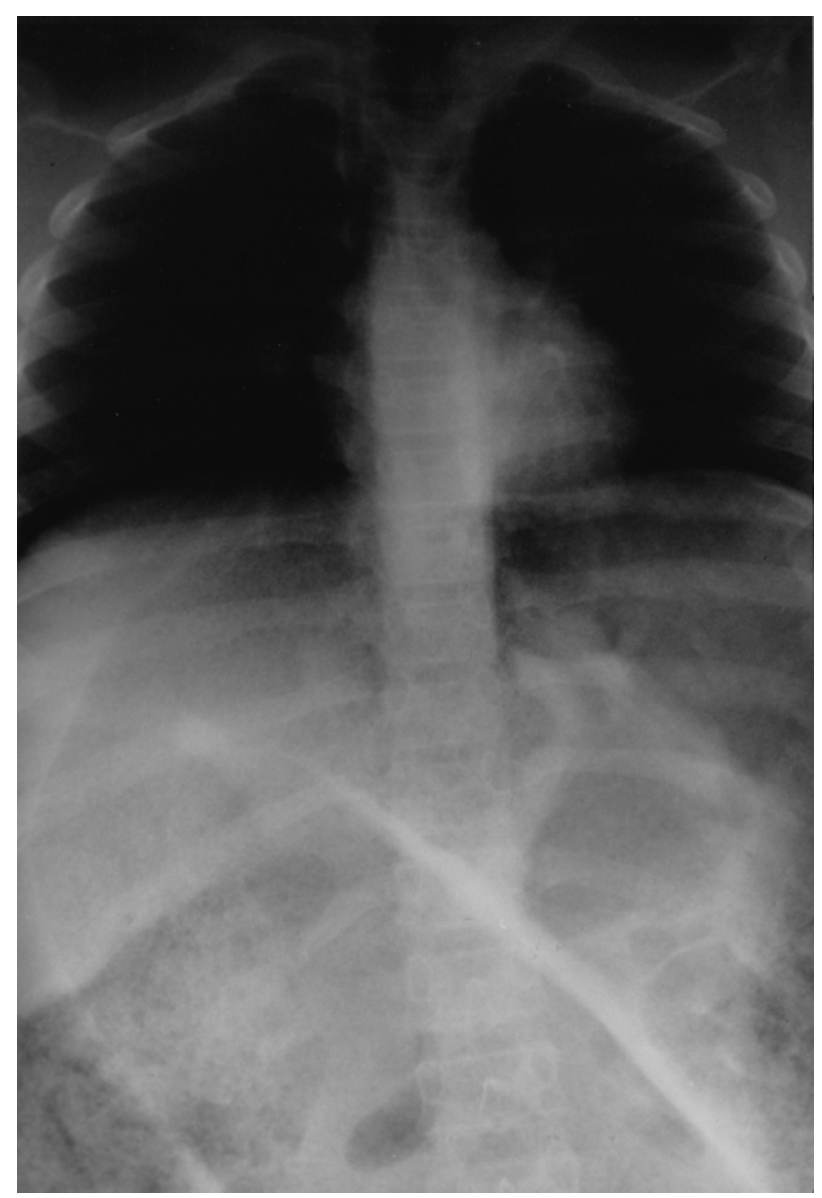

Fig 1. A preoperative abdominal radiograph shows an extremely dilated rectum and sigmoid colon. mately $5 \mathrm{~cm}$ above the anal verge. The dilated rectum was then resected, and a 1-layer, hand-sewn anastamosis was performed between the nondilated sigmoid colon and the remaining rectal stump.

The cecum was mobilized and, after appendectomy, a Bard Gastrostomy button (CR Bard, Inc, Covington, GA) was inserted through the stump and secured with 2 concentric pursestring sutures. The button then was brought through the abdominal wall via a separate stab incision, and the cecum circumferentially anchored at this site.

Postoperatively, feedings were withheld for 3 to 4 days; systemic antibiotics were continued for 72 hours. The cecostomy buttons gravity drained for the first 3 days and then clamped until postoperative day 14 when antegrade irrigations were started. Irrigation was performed daily with polyethylene glycol solution and volumes adjusted on an individual basis.

\section{RESULTS}

Constipation and incontinence resolved within 6 months in all patients. Furthermore, all children remained continent without the aid of laxatives, suppositories, or bulk-forming agents. At a mean follow-up of 37 months (15 to 60 months), there were no postoperative episodes of fecal impaction, and the only complication was 1 episode of antibiotic-associated diarrhea. Daily antegrade irrigations were administered through the cecostomy button and were accepted enthusiastically by both the patients and their parents. In 3 patients, the number of irrigations was decreased gradually and eventually discontinued. Their cecostomy buttons were removed subsequently at 1-year postplacement, and all 3 children have continued to maintain regular bowel habits. The fourth patient never required antegrade irrigations and also has had her cecostomy button removed at 1 year.

Three patients underwent repeat anorectal manometry, and all 3 patients showed normal rectal sensory threshold volumes and anorectal inhibitory reflexes. Barium studies also were obtained in 3 patients without evidence of recurrent rectal dilation (Fig 2).

\section{DISCUSSION}

Chronic, unremitting constipation and fecal soiling is a challenging problem to everyone involved in taking care of these children, ranging from family members to physicians. The conservative management of this debilitating condition includes initial clean out of the colon followed by long-term bowel regimens that often include the use of frequent rectal enemas. Katz et $\mathrm{al}^{9}$ reported that strict adherence of such long-term regimens led to a good or excellent result in $66 \%$ of patients with functional constipation. Despite such success, rectal enemas are frequently abandoned because of the difficulty and psychologically traumatic nature associated with implementing such procedures as the child grows older and begins to actively protest or resist. Older children, especially teenagers, find rectal enema administration to be tedious and inconvenient and would rather accept the 


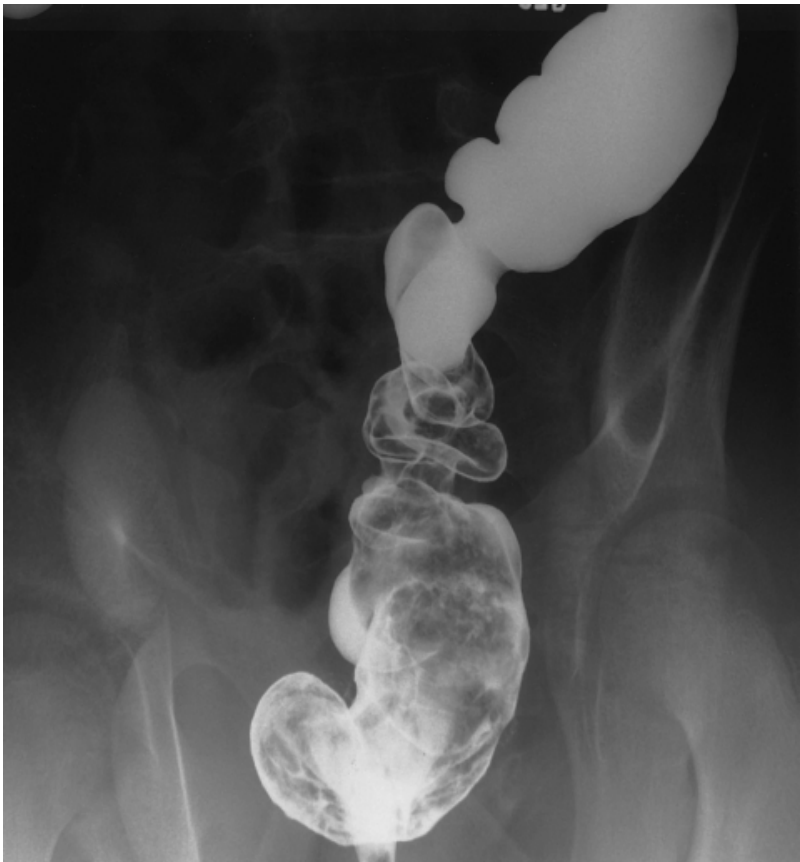

Fig 2. A postoperative barium enema shows normal caliber descending colon.

consequences of being constipated and incontinent. ${ }^{10}$ Thus, the actual number of patients treated successfully with medical therapy actually may be much less than that reported in the literature. Taking this into consideration, alternative approaches have been developed in the treatment of refractory constipation to achieve improved patient compliance. Antegrade enemas for fecal incontinence was first introduced by Malone in 1990. ${ }^{11,12}$ This concept dates back to 1968 when Muir ${ }^{13}$ first described the use of antegrade colonic washouts through cecostomy tubes as part of ontable colonic preparation before large bowel surgery. Since Malone's initial report of antegrade enemas by way of an appendicocecostomy conduit, multiple operative revisions have been described. ${ }^{5,10}$ Despite these modifications, the basic goal of antegrade enemas has remained the same and is similar to that of rectal enemas, which is to achieve total colonic evacuation and controlled continence.

In a specific subset of patients, however, chronic fecal retention and encopresis may persist even when initial colonic emptying and strict medical management is achieved. Often, these patients will have a massively dilated rectum that lacks sufficient sensory stimulus to initiate an anorectal reflex except at supranormal (and occasionally extraordinary) balloon volumes. Furthermore, the massively dilated rectum may show decreased propulsive function, thereby impeding evacuation. These 2 findings are associated commonly with each other, and the end result is a functional obstruction manifested by fecal retention, impaction, and soiling that is unrespon- sive to medical management. ${ }^{7}$ In such cases, isolated reports of segmental resection of the dilated segment of bowel has led to successful relief of symptoms. ${ }^{7,8}$

The association between poor bowel motility and extremely dilated segments of rectum after imperforate anus repair has been described previously. ${ }^{7,14}$ At birth, the wall of the dilated rectum in patients with preexisting anorectal malformations is thin walled and contains a normal (or smaller than normal) number and size of muscle cells. ${ }^{15}$ If this ectatic rectum is not resected at the time of reconstruction, it can result in a functional obstruction and pseudoincontinence. Secondary smooth muscle cell hypertrophy and hyperplasia then develop as a result of this distal obstruction. ${ }^{15}$ Thus, in patients with primary rectal ectasia, abnormal motility precedes severe constipation, impaction, and incontinence, which then lead to further dilation of the rectum.

Unlike the patients with primary rectal ectasia, patients with idiopathic megarectum (as described by this report) had normal initial bowel function with no underlying anatomic or neuromuscular disorder. After years of continued fecal retention, for whatever reason (rectal fissures, rectal trauma, behavioral disorders), megarectum developed which then led to impaired motility and further worsening constipation, fecal impaction, and encopresis. Initial anorectal manometry in all patients was abnormal, showing markedly increased sensory threshold volumes and diminished but present anorectal inhibitory reflex. Once the megarectum was resected, however, repeat anorectal manometry was performed in 3 patients, of which all were completely normal. Furthermore, constipation and incontinence resolved in all patients even after discontinuing the antegrade enemas. Barium enemas also showed no recurrence of the megarectum and correlated with normal bowel habits. Thus, it appears that in patients with idiopathic megarectum, impaired motility is a result of chronic retention and overstretching of the bowel, whereas the opposite is true in patients with primary rectal ectasia. Despite the cause, however, resection of the abnormally dilated rectum leads to improved or normal bowel function.

Cecostomy buttons were removed in all 4 patients 1 year after the initial procedure, and normal bowel habits continued. These findings question whether cecostomy buttons were needed in the first place. We initially elected to insert cecostomy buttons based on our positive experience using such devices in patients with bowel dysmotility syndromes and those with constipation and incontinence after surgically corrected anorectal malformations. Furthermore, the underlying pathoetiology resulting in fecal retention in patients with functional constipation is unknown and, quite possibly, varied. Therefore, we were expecting such children to have more difficulty in maintaining normal habits even after resec- 
tion of the megarectum and thus require antegrade enemas to relieve occasional bouts of constipation or fecal soiling. In short, our initial presumption was that use of the cecostomy button with antegrade enemas would be the primary, long-term management strategy in these patients, and the role of megarectum resection was to hasten the achievement of an effective cathartic regimen. The rapid reduction in the need for cathartic agents after megarectum resection suggests that the principal determinant for ongoing fecal retention in this patient population is the megarectum itself.

Currently, we still favor insertion of cecostomy tubes because they offer many advantages and minimal morbidity. Unlike access using an appendicocecostomy, cecostomy tubes are temporary and may be removed easily without requiring another operation. Using daily antegrade colonic enemas routinely achieves complete colonic evacuation, thus, psychologically preparing these children to have normal bowel habits. Given the few number of patients and limited follow-up in this preliminary experience, we are not yet ready to assume that megarectum resection alone is a predictable solution in all such children. Therefore, having a cecostomy tube in place safeguards against recurrent constipation and soiling after segmental resection.

This method of treatment is directed at a specific subset of patients. These patients must have no underlying organic disorder, have failed long-term medical management, and have evidence of megarectum on contrast enema. Once the diagnosis of megarectum is made, operative intervention should not be delayed because conservative management not only fails but also may have significant psychological and physical morbidity. Gattuso et al ${ }^{16}$ reported internal and external anal sphincter disruption in greater than $60 \%$ of patients undergoing manual disimpaction. Unlike treatment for constipation in adults, resection should be limited only to the grossly dilated segment of bowel to prevent fecal incontinence. ${ }^{17}$ Furthermore, as shown by the results of this report and those by Cheu and Grossfeld, ${ }^{7}$ preserving a rectal pouch as advocated by Peña and Behery ${ }^{8}$ is not necessary to prevent incontinence. Although not substantiated, we feel that leaving a poorly functioning pouch may predispose these patients to future recurrence of constipation and incontinence.

We recognize that the majority of patients with chronic functional constipation can be treated successfully with conservative management. However, surgical management may be required to treat refractory constipation and incontinence, especially when associated with megarectum. In this situation, we feel that proctectomy and button cecostomy for antegrade enemas is a safe and technically feasible treatment option. There were no associated intraoperative complications or deaths, and there was only one minor postoperative complication (Clostridium difficile colitis). Further follow-up is required to ensure that all patients remain continent and with normal bowel habits without the aid of medication.

\section{REFERENCES}

1. Fishman LN, Israel EJ: An approach to the child with constipation. Semin Colon Rectum 5:116-123, 1994

2. Clayden GS: Management of chronic constipation. Arch Dis Child 67:340-344, 1992

3. Simpson BB, Ryan DP, Schnitzer JJ, et al: Surgical evaluation and management of refractory constipation in older children. J Pediatr Surg 31:1040-1042, 1996

4. Fukunaga K, Kimura K, Lawrence JP, et al: Button device for antegrade enema in the treatment of incontinence and constipation. J Pediatr Surg 31:1038-1039, 1996

5. Redel CA, Motil KJ, Bloss RS, et al: Intestinal button implantation for obstipation and fecal impaction in children. J Pediatr Surg 27:654-656, 1992

6. Mishalany H: Seven years' experience with idiopathic unremitting chronic constipation. J Pediatr Surg 24:36-362, 1989

7. Cheu HW, Grosfeld JL: The atonic baggy rectum: A cause of intractable obstipation after imperforate anus repair. J Pediatr Surg 27:1071-1073, 1992

8. Peña A, El Behery M: Megasigmoid: A source of pseudoincontinence in children with repaired anorectal malformations. J Pediatr Surg 28:199-203, 1993

9. Katz C, Drongowski RA, Coran AG: Long-term management of chronic constipation in children. J Pediatr Surg 22:976-978, 1987
10. Shandling B, Chait PG, Richards HF: Percutaneous cecostomy: A new technique in the management of fecal incontinence. J Pediatr Surg 31:534-537, 1996

11. Malone PS, Ransley PG, Kiely EM: Preliminary report: The antegrade continence enema. Lancet 336:1217-1218, 1990

12. Graf JL, Strear C, Bratton B, et al: The antegrade continence enema procedure: Review of the literature. J Pediatr Surg 33:12941296, 1998

13. Muir EG: Safety in colonic resection. Proc R Soc Med 61:401408, 1968

14. Powell RW, Sherman JO, Raffensperger JG: Megarectum: Rare complication of imperforate anus repair and its surgical correction by endorectal pullthrough. J Pediatr Surg 17:786-795, 1982

15. Brent L, Stephens FD: Primary rectal ectasia. A quantitative study of smooth muscle cells in normal and hypertrophied human bowel. Prog Pediatr Surg 9:41-62, 1976

16. Gattuso JM, Phil M, Kamm MA, et al: The anal sphincter in idiopathic megarectum: Effects of manual disimpaction under general anesthetic. Dis Colon Rectum 39:435-439, 1996

17. Vasilevsky CA, Nemer FD, Balcos EG, et al: Is subtotal colectomy a viable option in the management of chronic constipation? Dis Colon Rectum 31:679-681, 1988 